\title{
Periodontal disease and respiratory disorders
}

\author{
K. Malathi*, S. Sujatha. \\ Professor \& Head, Department of Periodontics, Tamil Nadu government dental college \& hospital, Chennai - 03. \\ Post Graduate student, Tamil Nadu government dental college \& hospital, Chennai - 03. \\ *Corresponding author E-mail: malsmoni@gmail.com
}

\begin{abstract}
The oral cavity has a multitude of functions in relation to daily life. The poor health has the potential of affecting the quality of life. In recent years, efforts have been made to recognize oral health as an integral part of overall health. There is a fair evidence exists between respiratory disorders and poor oral hygiene \& good evidence exist that improved oral health and professional oral health care reduce the progression or occurrence of respiratory diseases among high risk adults. This paper discusses the relation between the periodontitis and its influence on the incidence of respiratory disorders.
\end{abstract}

Keywords: Respiratory disorders, chronic periodontitis, chronic obstructive pulmonary disorders, asthma, selective digestive decontamination.

\section{Introduction}

A chronic respiratory infection is a constant potential source of infection and has been considered as a separate risk factor for respiratory diseases. Evidences dictate a bi-directional relationship between periodontitis and respiratory diseases [1].

Periodontitis affects individuals of all ages but it is more common in elderly patients [2]. It is an infection of global prevalence and increased prevalence, extent, \& severity in older age groups depends on the exposure periods to the established risk factors like cigarette smoking [3].

The oral cavity has long been considered a potential reservoir for respiratory pathogens [4]. Scannapieco et al showed that lung functions decreased with increased periodontal attachment loss. Therefore they concluded that a potential association between periodontitis and chronic pulmonary diseases like chronic obstructive pulmonary diseases may exist [5].Evidences are there showing nearly fivefold increase in chronic respiratory diseases in subjects that had poor oral hygiene when compared to those with good oral hygiene[6].

In high risk groups, poor oral hygiene, periodontitis influence the incidence of pulmonary infections, especially in patients with episodes of nocsocomial Pneumonia. Several mechanisms are proposed to attempt to explain the potential role of oral bacteria in the process of respiratory infections [7].

\section{History}

The concept of focal infection has been known since antiquity. The term oral sepsis used by Hunter was replaced by the term focal infection in 1911 by Frank Billing, He defined Focus of infection as "circumscribed area of tissue infected with pathogenic organism ". Renewed attention has been focused on oral sepsis and its relationship with systemic factors. An old concept is being examined in new height.

Respiratory diseases are responsible for a significant number of deaths and considerable sufferings in human respiratory tract infections. Respiratory tract infections such as Pneumonia and exacerbation of chronic obstructive pulmonary diseases involve the aspiration of bacteria from the oropharynx into the lower respiratory tract [8]. 


\section{Chronic periodontitis}

Chronic periodontitis is an inflammatory disease caused by the plaque microorganisms, resulting in progressive destruction of tissues that support the teeth [9]. Periodontitis is characterized by periods of exacerbation with periods of remission. In chronic periodontitis there is increased local microbial burden that initiates local inflammation resulting in local tissue destruction.

\section{Dental considerations}

\subsection{Role of oral bacteria in respiratory infections:}

Teeth can serve as a reservoir for respiratory infections. Toxins released in the salivary secretions from microorganism's in the dental plaque, when aspirated in to the lower respiratory tract causes Pneumonia [10] [11]. It is already a known fact that severe anaerobic lung infections can occur following aspiration of salivary secretions, especially with periodontal diseases. Oral bacteria may also have a role in the exacerbation of Chronic Obstructive Pulmonary Disorders. Anaerobic bacteria were cultured from $17 \%$ of transtracheal aspirations from patients with chronic obstructive pulmonary disorders (COPD). The distal airways of chronic obstructive pulmonary disorders (COPD) subjects frequently show bacterial colonization by presumably nonpathogenic oral bacteria. Laboratory studies suggest that oral anaerobes such as Porphyromonas gingivalis can cause marked inflammation when installed in to the lungs of lab animals [7]. A relationship between the systemic humoral response to Prevotella species which is associated with periodontal diseases and ventilator associated Pneumonia was discussed by Groiller et al [12].

\subsection{Dental plaque - Reservoir of respiratory pathogens:}

Lack of attention to oral hygiene can result in an increase in the mass and complexity of dental plaque, which may foster bacterial interactions between indigeneous plaque bacteria and respiratory pathogens such as P. aeruginosa and enteric bacilli [13]. These interactions may result in colonization of dental plaque by respiratory pathogens. Respiratory pathogens that establish in dental plaque may be difficult to eradicate. Respiratory pathogens also colonize in the oral cavity of patients with teeth or denturers than edentulous patients not wearing dentures [14].

\section{Etiopathology}

Periodontitis is an infective condition attributable to certain pathogens, which causes destruction in association with inflammatory mediators. The mechanism of destruction involves both direct tissue damage resulting from plaque bacterial products and indirect damage through bacterial induction of the inflammatory \& immune responses [15].

\section{Mechanism of action of oral bacteria in the pathogenesis of respiratory diseases}

The mechanism of action could be aspiration of oral bacteria, colonization of dental plaque by respiratory pathogens causing Pneumonia into the lungs [2], [16]. 
Other mechanism include, Alteration of the mucosal surface by salivary enzymes in periodontitis

Leading to an increase in adhesion

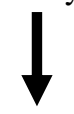

(Colonization)

Colonization of respiratory pathogens

\section{(Enzymatic destruction)}

Destruction of salivary pellicles on pathogenic bacteria, by periodontal disease associated enzymes Cytokines:

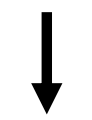

Facilitating infiltration of the epithelium by respiratory pathogens.

\section{Mechanism of tissue destruction in periodontitis}

PERIODONTAL TISSUE

Pathogenic bacterial flora

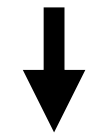

Release of bacterial proteases

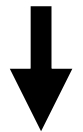

Activation of components

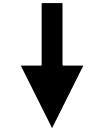

Recruitment \& activation of neutrophils

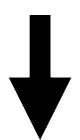

Frustrated phagocytosis

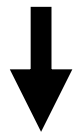

Release of neutrophil proteinases

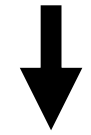

Abnormal connective tissue destruction

Periodontitis Emphysema

\section{LUNG}

foreign material

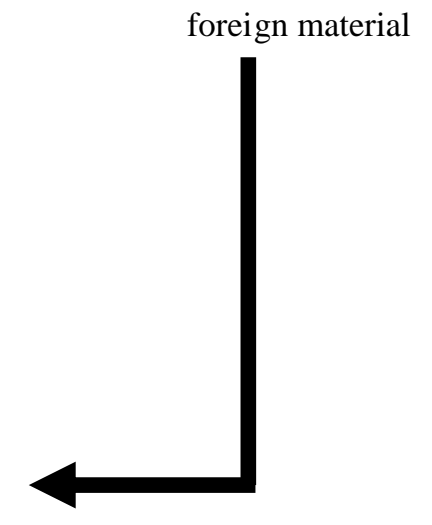


According to Travis et al (1994) Pulmonary emphysema and Periodontitis share a similar mechanism of tissue destruction. In Periodontitis \& Emphysema degranulation of neutrophil occurs during attempted phagocytosis (so called frustrated phagocytosis) which release proteolytic enzymes. Destruction of the pulmonary alveoli and the periodontal attachment results due to degradation of proteins from connective tissues [4] [17].

\section{Asthma and periodontal disease}

Asthma is characterized by recurrent episodes of wheezing, shortness of breath, chest tightness and cough , during recovery there may be pus like material comes out due to the presence of high levels of eosinophils in the in the white blood cells. Association between asthma and periodontal disease may involve activation of the immune system due to inflammatory nature of the both. Serum Ig A secreted in saliva act as a first line of defense for the mucosa and has a major role in restricting the periodontal diseases.

Studies have revealed reduction of serum Ig in saliva of asthmatics. Destruction of periodontium found to be higher in asthmatics than in non-asthmatics according to Periodontal Disease Index (PDI). Similarly gingival Ig E concentrations in asthmatics found elevated in patients with periodontal diseases [18].

\section{Dent bronchial syndrome}

Syndromes affecting the upper respiratory tracts have a link between periodontitis and productive cough. Relation between productive cough, post nasal drip and periodontitis affecting the teeth near maxillary sinus can lead to the dent bronchial syndrome threefold as that of in general population out of which the prevalence of cough and periodontitis has been markedly increased in smokers who had smoked for several years [19].

\section{Prevention of oral colonization by potential respiratory pathogens}

Studies have documented a shared association between periodontal disease and an increased frequency of oral infections. Studies have evaluated the role of poor oral hygiene and periodontal diseases in the development of pneumonia in high risk patients. Trepanning et al observed an association between the development of aspiration Pneumonia and dental status. The improved oral hygiene in the hospital setting may decrease the occurrence of or pharyngeal colonization by respiratory pathogens and thus decrease the risk of Nosocomial Pneumonia [20]. Current oral hygiene measures are probably inadequate to prevent dental plaque formation.

\subsection{Selective digestive decontamination (SDD):}

This method uses antibiotics. Topically applied antibiotics to the surfaces of the GIT, including the oral cavity reduces the carriage of pathogenic bacteria and thus to respiratory infections [21]. The use of lozenges containing Polymyxin-B, Tobramycin and Amphotericin-B has been shown to diminish oral colonization of gram negative bacilli [22]. However SDD does not appear to have an effect on the mortality rate and seems to foster the selection of antibiotic resistant bacteria and cross infection. Focusing specially on factors on promoting oropharyngeal bacterial colonization may be useful in other developing strategies to prevent colonization and thereby prevent bacterial Pneumonia in high risk population.

Maintenance of good oral hygiene by itself reduces oropharyngeal colonization by respiratory pathogens. Chlorhexidine appears to be a reasonable choice for maintaining good oral hygiene to reduce plaque and salivary levels of bacteria by up to $85 \%$ [23]. Transfer of group B Streptococcus from mother - infant during parturism is reduced due to the usage of chlorhexidine gluconate [24]. An analogous method used the mouth may inhibit oral colonization by respiratory pathogens, with minimal risk. Chlrorhexidine appears to inhibit the production of proteases by subgingival bacteria. By inhibiting the proteases activity, chlorhexidine may diminish the potential of these enzymes to precede oral surfaces to expose "cryptitopes" [25]. 


\section{Conclusion}

Periodontal diseases may influence the course of respiratory infections. Aspiration of oral pathogens into the lungs is the main cause of respiratory infections. Other than the enzymes in the saliva, cytokine originating from periodontal tissue are the different other mechanism envisioned to explain how oral bacteria can participate in the pathogenesis of respiratory infections. It is also possible that periodontitis might not be directly related to the causation of this systemic condition. But both of these might share common risk factors. They have potential pathogenic organisms in common. Rather than periodontal diseases being confined only to periodontium, periodontal diseases may have a wide range of systemic effects. Hence the dentist will need to assume larger responsibilities for the overall health of the patients.

Number of recent microbiologic and epidemiologic studies suggests a relationship between poor oral health and respiratory diseases, especially in high risk subjects. It is conceivable that improved oral hygiene may decrease the prevalence of oropharyngeal colonization by respiratory pathogens and thereby reduce the risk of infection in high risk subjects. This suggests that the Periodontist must be prepared to provide safe and effective therapy to patients and helping them to prevent the occurrence of various systemic involvements from the poor oral hygiene.

\section{References}

[1] Role of Oral bacteria in Respiratory infections-Journal of Periodontology 1999 vol.70; pg 793-802.

[2] Bently DW .Bacterial pneumonia in the elderly: clinical features, diagnosis, etiology, and treatment. Gerontologist 1984; $30: 297-307$.

[3] Hospital acquired pneumonia in adults: diagnosis, assessment of severity, initial antimicrobial therapy, and preventive strategies. A consensus statement, American Thoracic Society, November 1995. Is J Respir Crit Care Med. 2001; 163:1730-1754?

[4] Majon P. Oral health and respiratory infection. J Can Dent Assoc. 2002:68:340-345.

[5] Scannapieco FA Potential association between chronic respiratory diseases and periodontal diseases. Analyses of National Health and Nutritional Examination Survey III Journal of Periodontology 2000 71; 1528-34.

[6] Hayes C.Sparrow D, Cohen M et al. The association between alveolar bone loss and pulmonary infections: The VA Longitudinal Study. Ann. Periodontology. 1998; 3:257-261.

[7] Periodontal Medicine Louis F.Rose, Robert J.Genco pg 83-97.

[8] Frank A. Scannapieco, Michael P.Rethman. Relationship between periodontal diseases and respiratory diseases. August 2003.

[9] Carranza's Clinical Periodontology, 10th edition pg 103-104.

[10] Periodontology 2000 Volume 44, 2007 pg164-177.

[11] Finegold S.M. Aspiration pneumonia Rev Infec Diseases 1991; 13:s737-42.

[12] Grollier G, Dore P, Robert R, et al. antibody response to Prevotella spp. in patients with ventilator associated pneumonia. Clin Diag Lab Immunol 1996; 3:61-65.

[13] Komiyama K, Tynan JJ, Habbick BF,et al. Pseudomonas aeruginosa in the oral cavity and sputum of patients with cystic fibrosis. Oral Surg Oral Med Oral Pathol 1985; 59:590-594.

[14] Scannapieco FA. Role of oral bacteria in respiratory infections. J Periodontology. 1999; 70:793-802.

[15] Carranza's Clinical Periodontology, 10th edition pg 120-121.

[16] Scannapieco FA, Stewart EM, Mylotte JM. Colonization of dental plaque by respiratory pathogens in medical intensive care patients. Crit Care Med 1992; 20; 740-745.

[17] Khair OA, Davies RJ, Devalia JL. Bacterial induced release of inflammatory mediators by bronchial epithelial cells. Eur Respir J 1996; 9:1913-22.

[18] S. Yaghobee, M. Paknejad. Journal of Dentistry, Tehran University of Medical Sciences, 2008; vol: 5 no.2.

[19] Christian O. Brandum, Bjarne Klausen Periodontology 2004; vol 1, issue 1: 43-49.

[20] Terpenning M, Bretz W, Lopatin D, et al. bacterial colonization of saliva and plaque in the elderly. Clin Infect Dis. 1993; 16(suppl 4):s314s316.

[21] Kerver AJH, Rommes JH, Mevissen-Verhage EAE, et al. prevention of colonization and infection incriticalli ill patients: a prospective randomized study. Crit Care Med 1988; 16:1087-93.

[22] Spijkerver FKL, Saene HKFV, Saene JJMV, et al. effect of selective elimination of the oral flora on mucositis in irradiated head and neck cancer patients. J Surg Oncol 1991; 46:167-73.

[23] Balbuena L, Stambaugh KI, Ramirez SG, Yeager C. Effects of topical oral antiseptic rinses on bacterial counts of saliva in healthy human subjects. Otolaryngology Head Neck Surg 1998; 118:625-629.

[24] Nilsson G, L arson L, Christensen K, et al. Chlorhexidine for prevention of neonatal colonization with group B streptococci. V. chlorhexidine concentrations in blood following vaginal washing durion delivery. Eur J Obstet Gynec Reprod Biol 1989;310:221-6

[25] Gibbons RJ, Hay DI, Childs WC, Davis G. Role of cryptic receptors(cryptitopes) in bacterial adhesion to oral surfaces. Arch Oral Biol 1990; 35:107S-114S. 